\title{
Micropatterned Scaffolds with Immobilized Growth Factor Genes Regenerate Bone and Periodontal Ligament-Like Tissues
}

\author{
Sophia P. Pilipchuk, Tobias Fretwurst, Ning Yu, Lena Larsson, Nolan M. Kavanagh, \\ Farah Asa'ad, Kenneth C. K. Cheng, Joerg Lahann, and William V. Giannobile**
}

Periodontal disease destroys supporting structures of teeth. However, tissue engineering strategies offer potential to enhance regeneration. Here, the strategies of patterned topography, spatiotemporally controlled growth factor gene delivery, and cell-based therapy to repair bone-periodontal ligament (PDL) interfaces are combined. Micropatterned scaffolds are fabricated for the ligament regions using polycaprolactone (PCL)/polylactic-co-glycolic acid and combined with amorphous PCL scaffolds for the bone region. Scaffolds are modified using chemical vapor deposition, followed by spatially controlled immobilization of vectors encoding either platelet-derived growth factor-BB or bone morphogenetic protein-7, respectively. The scaffolds are seeded with human cells and delivered to large alveolar bone defects in athymic rats. The effects of dual and single gene delivery with and without micropatterning are assessed after 3, 6, and 9 weeks. Gene delivery results in greater bone formation at three weeks. Micropatterning results in regenerated ligamentous tissues similar to native PDL. The combination results in more mature expression of collagen III and periostin, and with elastic moduli of regenerated tissues that are statistically indistinguishable from those of native tissue, while controls are less stiff than native tissues. Thus, controlled scaffold microtopography combined with localized growth factor gene delivery improves the regeneration of periodontal bone-PDL interfaces.

\section{Introduction}

Current clinical therapies do not predictably reconstruct the bone-ligament interfaces of tooth-supporting tissues damaged by trauma or periodontal disease. The regeneration of periodontal tissue supporting structures requires the consideration of specific anatomical architectures. The alveolar bone-periodontal ligament (PDL)-cementum complex consists of alveolar bone and bundle bone that houses ligamentous Sharpey's fibers; these fibers anchor the PDL to the cementum, a mineralized layer that covers the tooth root. ${ }^{[1]}$ The development of biomaterial systems for regenerating these complex tissues must necessarily consider the scaffold material, shape, and topography, as well as the inclusion of growth factors and cells.

Several studies have examined scaffold designs and growth factors that support multitissue regeneration relevant to periodontal tissue engineering. ${ }^{[2]}$ Material surface topography, such as roughness, is an
Dr. S. P. Pilipchuk, Dr. T. Fretwurst, ${ }^{[+]}$Dr. N. Yu, Prof. L. Larsson,

N. M. Kavanagh, Dr. F. Asa'ad, Prof. W. V. Giannobile

Department of Periodontics and Oral Medicine

University of Michigan School of Dentistry

1011 N. University Ave., Ann Arbor, MI 48109, USA

E-mail:wgiannob@umich.edu

Dr. S. P. Pilipchuk, Prof. J. Lahann, Prof. W. V. Giannobile

Department of Biomedical Engineering

University of Michigan College of Engineering

1101 Beal Ave., Ann Arbor, MI 48109, USA

Prof. L. Larsson

Department of Periodontology

Institute of Odontology

Medicinaregatan $12 \mathrm{~F}$

University of Gothenburg

SE-405 30 Gothenburg, Sweden
Dr. F. Asa'ad

Institute of Odontology

Medicinaregatan 12F

The Sahlgrenska Academy at University of Gothenburg

SE-405 30 Gothenburg, Sweden

Dr. K. C. K. Cheng, Prof. J. Lahann, Prof. W. V. Giannobile

Biointerfaces Institute

Department of Materials Science and Engineering

University of Michigan College of Engineering

2800 Plymouth Rd, Ann Arbor, MI 48109, USA

Prof. J. Lahann

Department of Chemical Engineering

Department of Macromolecular Science and Engineering

University of Michigan College of Engineering

2800 Plymouth Rd, Ann Arbor, MI 48109, USA

The ORCID identification number(s) for the author(s) of this article can be found under https://doi.org/10.1002/adhm.201800750.

${ }^{[+]}$Present address: Department of Oral and Maxillofacial Surgery,

Medical Center - University of Freiburg, Faculty of Medicine, University

of Freiburg, Hugstetter Straße 55, Freiburg D-79106, Germany

DOI: 10.1002/adhm.201800750 
important parameter in determining cellular response. ${ }^{[3]}$ It has been shown that fibroblasts have a preference for attaching to smooth surfaces, ${ }^{[4]}$ while bone-derived cells prefer surfaces that are rough. ${ }^{[5]}$ In terms of organized microtopography, grooves of $10 \mu \mathrm{m}$ width have been shown to increase the adhesion and viability of osteoblastic cells..$^{[5,6]}$ As such, micropatterned scaffolds have been developed that incorporate grooves in the PDL region to mimic the oblique orientation of native PDL. This design was shown to promote the alignment of individual cells and subsequent collagenous tissue formation in vivo. ${ }^{[7]}$ Regarding growth factors, bone morphogenetic protein-7 (BMP-7) is a growth and differentiation factor important for stimulating the migration, angiogenesis, and differentiation of mesenchymal stem cells into bone-forming cells and cartilage. ${ }^{[8]}$ Platelet-derived growth factor (PDGF) has been shown to promote the regeneration of alveolar bone, PDL, and cementum. The isoform PDGF-BB, in particular, has been shown to safely and effectively treat periodontal osseous defects. ${ }^{[8,9]}$

Gene therapy-based growth factor delivery in combination with scaffolds has been suggested as a promising therapeutic approach for periodontal regeneration. ${ }^{[10]}$ These vectors can be delivered via adenoviruses. In a recent study, the use of a mesoporous bioglass (MBG)/silk fibrin scaffold delivering adenoviruses encoding the gene for BMP-7 (AdBMP-7) and others encoding PDGF-B (AdPDGF-B) promoted periodontal regeneration in periodontal defects. ${ }^{[11]}$ Similar results were reported for chitosan/collagen scaffolds that delivered AdBMP-7 or AdPDGF-B. ${ }^{[12]}$

Despite the successful use of gene therapy in combination with scaffolds for periodontal regeneration, a major limitation of using gene therapy vectors has been the lack of spatiotemporal control of gene expression. This concerns potential vector dispersion. To address this limitation, Lahann et al. developed chemical vapor deposition (CVD)-based polymer coatings that use antibodies to tightly immobilize gene therapy vectors onto FDA-approved biomaterials, including polycaprolactone (PCL) and polylactic-co-glycolic acid (PLGA). ${ }^{[13,14]}$ The CVD technique may represent an innovative method for targeting the effects of gene therapy to specific tissues or regions. ${ }^{[15-17]}$ In fact, surface modification using polymer coatings represents an effective means of delivering gene therapy vectors. Adenoviral vectors have been shown to efficiently deliver growth factor genes that are expressed over finite periods of time. ${ }^{[18]}$ To mitigate random release and systemic dispersion, the CVD method covalently immobilizes the adenoviruses onto the material's surface via antigen-antibody interactions. Moreover, by adjusting the amount of vector attached to the surface and its site of localization, CVD provides a way to establish a biological signaling gradient. ${ }^{[16]}$

In a model relevant to periodontal regeneration, Hao et al. showed that CVD-immobilized AdPDGF-BB and AdBMP-7 could efficiently transduce seeded human PDL cells and produce considerable levels of PDGF-BB and BMP-7 protein in vitro. ${ }^{[14]}$ In addition, the combination of gene delivery with 3D-printed scaffolding, wherein one scaffold region had immobilized AdBMP-7 and the other had AdPDGF-BB, demonstrated compartmentalized gene expression.

Therefore, the present study aimed to evaluate the combined effects of growth factor gene delivery on micropatterned scaffolds to regenerate periodontal defects in vivo. Micropatterned, PLGA/PCL scaffolds were designed to regenerate ligamentous structures and amorphous PCL scaffolds were designed to regenerate bone. Using the CVD technique, the former were immobilized with AdPDGF-BB, and the latter with AdBMP-7. The scaffolds were then seeded with human fibroblasts and implanted into large osseous fenestration defects, and their ability to regenerate bone and ligamentous tissue was evaluated at 3,6 , and 9 weeks.

\section{Results and Discussion}

\subsection{Design of Patterned Scaffolds and Spatially Controlled Immobilization of Gene Therapy Vectors}

The present study aimed to evaluate how the combination of scaffold topography and growth factor gene delivery could enhance periodontal tissue repair. The experimental treatment consisted of a biphasic scaffold, having a micropatterned, PLGA/PCL region that delivered AdPDGF-BB to promote PDL tissue regeneration, and an amorphous, PCL region that delivered AdBMP-7 to promote bone regeneration (Figure 1A). Here, the micropatterned pillars had grooves of $15 \mu \mathrm{m}$ width and $30 \mu \mathrm{m}$ depth, which were based on previously established parameters shown to promote the formation of aligned collagenous tissue. ${ }^{[7]}$ Grooved pillars were oriented toward the tooth root and designed to align newly formed ligamentous tissue in the oblique orientation of native PDL. The bone region was placed directly on top. The scaffold regions were each $250 \mu \mathrm{m}$ thick, for a combined thickness of $500 \mu \mathrm{m}$, providing a calculated fit within a $500 \mu \mathrm{m}$ deep, osseous fenestration defect (Figure 1). Adenoviruses were immobilized onto the scaffold surfaces using CVD to locally deliver gene vectors. Other groups included the single delivery of AdBMP-7 on both regions of the scaffold, which was selected based on a previous publication indicating that treatment of periodontal defects with ex vivo gene transfer of BMPs only resulted in cementogenesis, bridging of periodontal bone defects, as well as evidence of initial PDL-like tissue formation. ${ }^{[10]}$ The negative controls delivered no genes (Ad-empty vectors) with or without a micropatterned PDL region. This pattern may have resulted in an osteoconductive effect, which would explain the ingrowth of bone even for the patterned group devoid of the gene delivery. The pattern may have even been more osteoconductive than the amorphous bone region, which was designed to be osteoconductive.

In the present study, scanning electron microscopy (SEM) images of CVD-coated scaffolds confirmed the micropatterned architecture and porosity of the scaffold (Figure 1B), as well as the immobilization of adenovirus particles on its surfaces (Figure 1C). Previously, it was shown that viral particles can be immobilized with even distribution on various surfaces, including nonporous PLGA and PCL. ${ }^{[19]}$ In this study, the virus was confirmed to be immobilized onto the porous PCL (Figure 1C) and patterned PCL/PLGA (Supplementary Figure 3) compartments. To further investigate cell behavior and alignment on the patterned films, human periodontal ligament (hPDL) cells were seeded on the films for $72 \mathrm{~h}$, fixed, and 

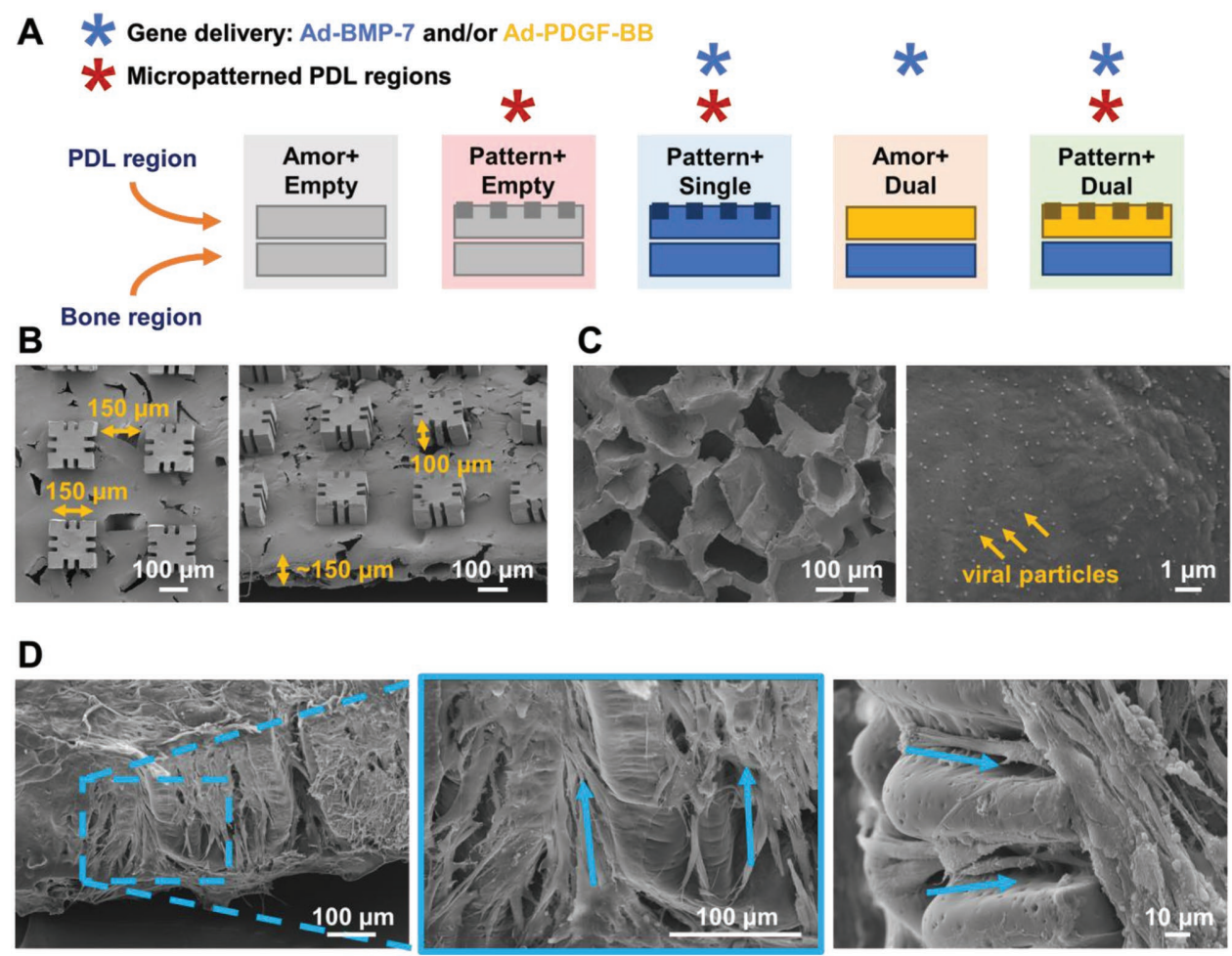

Figure 1. Scaffold design for periodontal tissue neogenesis. A) Schematic representation of the polymeric scaffolds, designed to deliver gene therapy vectors and/or micropatterned surface topography to the bony defects. The PDL regions were either patterned or amorphous, made of PLGA/PCL, and seeded with human periodontal ligament cells (hPDLs), except Pattern+Single, which received hGFs. The bone regions were amorphous, made of PCL, and seeded with human gingival fibroblasts (hGFs). All regions were CVD-coated to immobilize adenoviral genes for Ad-BMP-7 (blue), Ad-PDGF-BB (yellow), or Ad-empty (gray). B) SEM images of patterned PDL region, showing the pillar and groove dimensions. C) SEM images of CVD-coated, PCL, porous base with immobilized adenoviral particles $\left(10^{12} \mathrm{PN} \mathrm{mL}^{-1}\right.$ ). D) SEM images of hPDLs aligned with the micropatterning, $3 \mathrm{~d}$ after seeding. Blue arrows indicate the alignment of cells along the grooves embedded within the scaffold pillars, as well as in the interpillar regions.

analyzed using SEM. Images showed cell alignment along the pillar grooves as well as at the interpillar spaces (Figure 1D). Together, these findings indicate that the cells seeded onto the CVD-treated surface intended to promote their transduction with the adenoviral vector are induced to align due to the presence of incorporated micropatterns within the polymer surface, with the intention of sustaining this alignment as the cells continue to proliferate. Therefore, part of this study's goal was to incorporate these patterned, cell-seeded constructs into a fenestration defect to observe their ability to support cell alignment and subsequent tissue regeneration over the long term.

\subsection{Osseous Defect Creation and New Bone Formation}

Fenestration defects of $2 \mathrm{~mm} \times 3 \mathrm{~mm}$ were surgically created in the animals' mandibulae, removing not only the alveolar bone but also the cementum and other soft tissue structures (Supporting Information, Figure 1A). The scaffolds were placed inside and evaluated for their ability to regenerate the boneligament interface after 3, 6, and 9 weeks in vivo.

Microcomputed tomography (Micro-CT) scans were performed at three and six weeks postimplantation to evaluate new bone formation. At three weeks, considerably greater bone regeneration was observed for groups with growth factor gene delivery, compared to those with Ad-empty vector (Figure 2A), where the root largely remained exposed. These differences manifested in significantly greater bone volume for all groups with gene delivery relative to Ad-empty at three weeks (Figure 2B). There were no differences between the groups with gene delivery, suggesting that the single delivery of BMP-7 provided no advantage in bone formation over the dual delivery of BMP-7 and PDGF-BB. The percentage of the defect filled with new bone was also greater for two of the three gene delivery groups relative to Ad-empty, particularly for Pattern+Single and Amor+Dual (Figure 2C).

By six weeks, all groups except for the negative control demonstrated nearly complete bone coverage of the tooth roots. Differences in the bone volume between groups largely abated, except for greater volume for Amor+Dual relative to the negative control (Figure 2B). By six weeks, enough time had passed for self-repair capacity. However, two of the three gene delivery groups, including the experimental group, did maintain significantly greater bone fill percent than the negative control (Figure 2C).

Tissue mineral density (TMD), a measure of mineralized tissue maturity and measured by micro-CT, showed little variation between groups at three or six weeks, save for greater TMD for the negative control relative to the single gene delivery at three weeks (Figure 2D).

Of note, cementum regeneration was minimally observed in the present study. Specifically, regions which are expected 
A

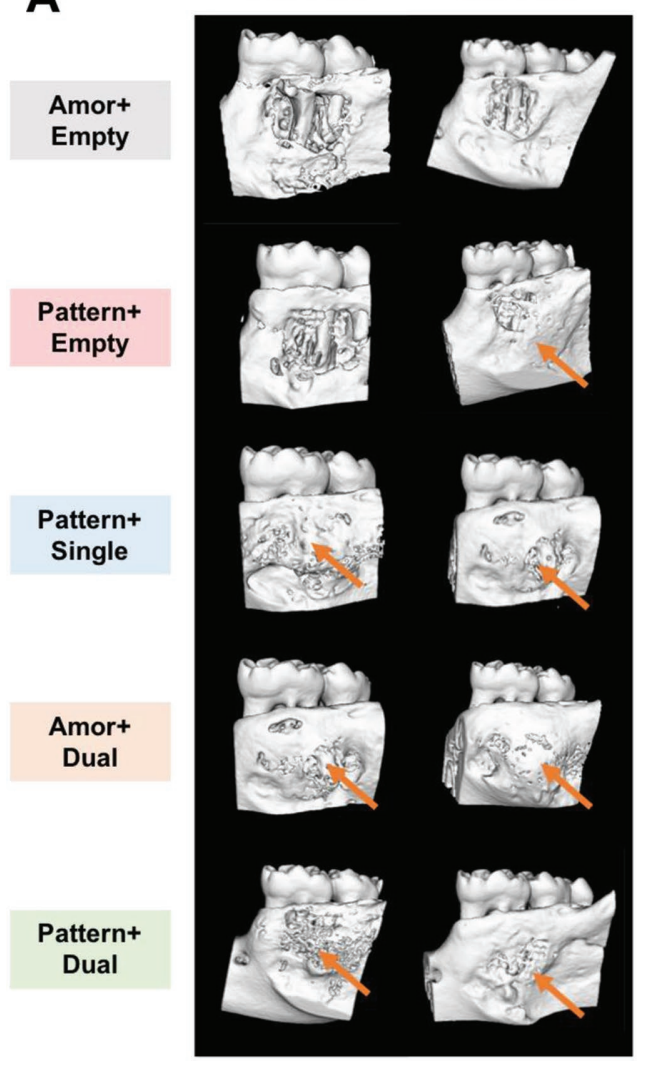

B

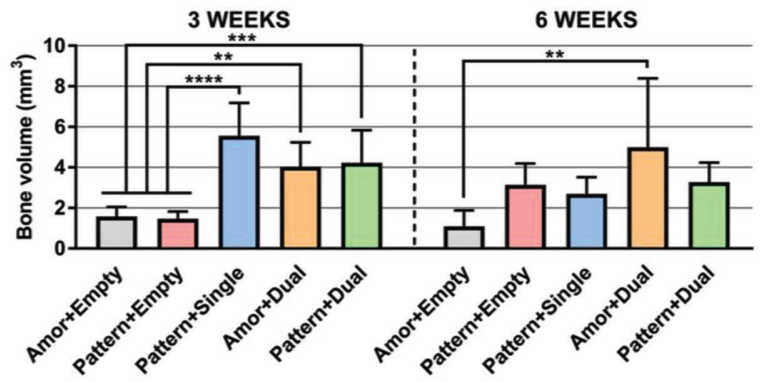

C

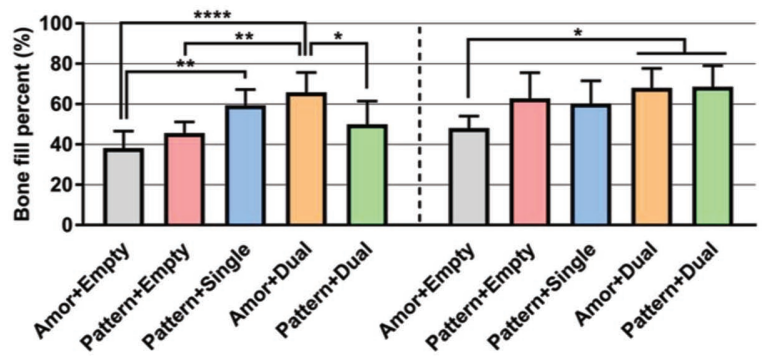

D

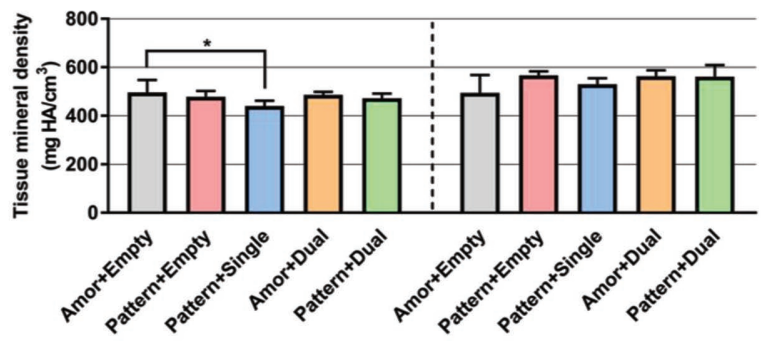

Figure 2. Micropatterned scaffolds and gene therapy promote new bone formation. A) Micro-CT isosurface images show the regenerated bone in the fenestration defects after three and six weeks for representative samples of each group. Arrows point to areas of considerable root coverage by new bone. B) New bone volume after three and six weeks. C) Percentage fill of the defect $(n=6-10)$. D) Tissue mineral density of the new bone ( $n=6-10$ animals per group). Data shown as mean \pm SD. Statistics performed as one-way ANOVA with Tukey's post hoc comparisons within each time point and two-sided $p$-values of * $p<0.05, * * p<0.01, * * * p<0.001, * * * * p<0.0001$

to display cementum formation at times appear as gaps between the tooth root surface and PDL-like tissue formation (Figure 3A) due to artifactual separation during histological processing, given that there is a lack of Sharpey's fiber formations that can stabilize the PDL-like tissue in place. Overall, while this study shows a lack of cementum formation which is necessary in periodontal tissues, further improvements in scaffold design are likely to improve this outcome, although formation of the cementum-PDL tissue complex is a challenge in numerous models. Intriguingly, Sowmya et al. recently were able to promote the formation of cementum using a trilayered nanocomposite hydrogel scaffold to deliver cells and growth factors to rabbit maxillary periodontal defects. ${ }^{[20]}$ Each layer targeted the regeneration of cementum (made of chitin-PLGA/nano-bioactive glass ceramic $[\mathrm{nBGC}]$ and delivered cementum protein 1$)$, PDL (chitin-PLGA with FGF-2), or alveolar bone (chitin/PLGA/ nBGC with platelet-rich plasma growth factors). Implantation of the scaffold with growth factors resulted in complete closure of the defect, tissue healing, and confirmed regeneration of cementum, fibrous PDL, and alveolar bone at one and three months-better than scaffold alone. These results may indicate that a separate growth factor or scaffold compartment may be more beneficial in order to more predictably repair cementum and ensure the formation of the full, trilayer periodontal complex.

\subsection{PDL-Like Tissue Formation, Assessed by Histology and Immunofluorescence}

The formation of ligamentous soft tissue was evaluated at three and six weeks postimplantation using histology. Hematoxylin and eosin (H\&E) staining showed the formation of soft tissue for all groups by three weeks (Figure 3A). However, the tissue of groups with patterned scaffolds was more likely to be aligned obliquely with the tooth root, like native PDL. By six weeks, this was even more so: the newly formed soft tissue of patterned groups, as well as Amor+Dual, had generated more of an oblique angle, while the negative control had not.

Toluidine blue staining was used to measure the mean width of the new soft tissue (Figure 3B). Interestingly, only the soft tissue of patterned groups closely approximated the width of native rat $\mathrm{PDL}$, which was $\approx 100 \mu \mathrm{m}$ (Figure $3 \mathrm{C}$ ). At three weeks, the widths for the three patterned groups were statistically indistinguishable from $100 \mu \mathrm{m}$, while those for the amorphous groups well exceeded $200 \mu \mathrm{m}$ and were significantly 
A
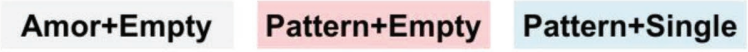

Amor+Dual

Pattern+Dual

3 WEEKS
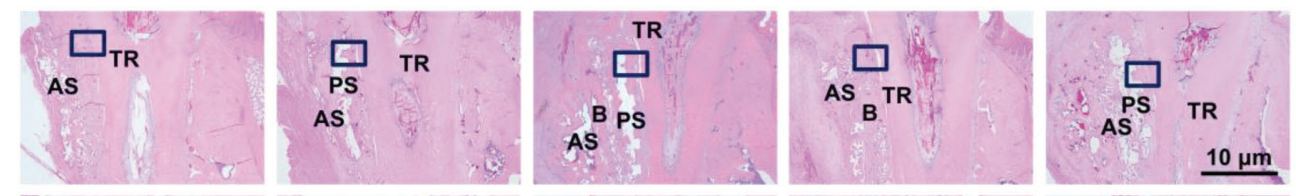

$40 X$
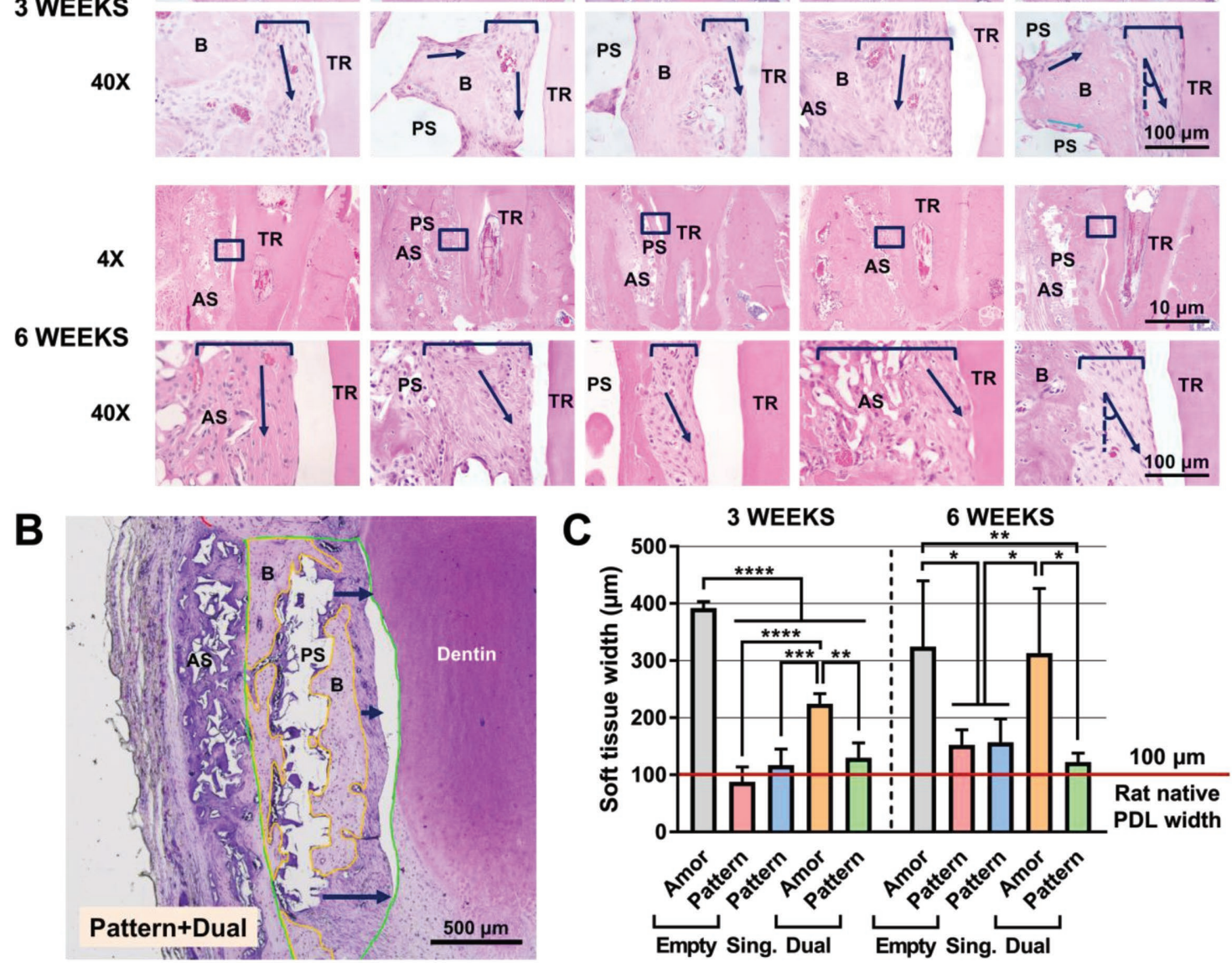

Figure 3. Micropatterned scaffolds with gene delivery promote ligamentous-like tissues in vivo. A) Hematoxylin and eosin staining shows the soft tissue formation at the defect site. Arrows indicate the general orientation of cell nuclei relative to the tooth root. Brackets indicate the soft tissue region, and boxes indicate the magnified regions in the images below. TR $=$ tooth root, $A S=$ amorphous scaffold, $P S=$ patterned scaffold, and $B=$ bone. B) Toluidine blue staining was used to measure soft tissue width. A representative slide of Pattern+Dual is shown. Blue arrows represent the three measurements averaged to determine the soft tissue width, the region of the total initial defect is outlined in green, and the region of new bone formation (B) is outlined in yellow. C) Measurements of soft tissue width from toluidine blue staining ( $n=3-6$ per group). Data shown as mean \pm SD. Statistics performed as one-way ANOVA with Tukey's post hoc comparisons within each time point and two-sided $p$-values of $* p<0.05$, $* * p<0.01$,

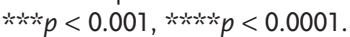

greater than those of all three patterned groups. At six weeks, the same result held, although the $95 \%$ confidence intervals for Pattern+Empty and Pattern+Single exceeded $100 \mu \mathrm{m}$. The micropatterning resulted in the generation of soft tissue possessing widths more consistent with native PDL. As mentioned earlier, the effects of surface topography may also explain the formation of new bone in front of the patterned scaffolds (Figure 3B). In effect, this result is contradictive to the scaffold design, within which the pattern is intended to promote aligned tissue formation. However, it is interesting to note that nonetheless there is presence of PDL-like tissue alignment in front of the bone region infiltrating into the interpillar regions, indicating that the osteoconductive nature of the patterning is not necessarily a counterproductive result to the regenerative process, and may in fact contribute to the resulting tissue alignment. This is likely due to the expression of BMP-7 by gingival fibroblasts transduced by AdBMP-7 on the PCL porous base region of the scaffold, resulting in increased expression of the growth factor and its presence in scaffold regions of the defect not intended for the stimulation of bone formation. At the same time, the presence of the patterning introduces another preferable condition for bone formation, specifically the presence of pillars that act as anchors for osseous tissue formation in an osteoconductive manner. While this was a surprising finding here, further investigation of the literature confirms that such an effect does exist, with studies showing that presence of microstructures in titanium (Ti)-based bone substitutes resulted in excellent osteoconduction. ${ }^{[21]}$ 
Likewise, incorporation of microstructures onto Ti implant surfaces, such as nano/micro nest-like and nanotubes structures, has been shown to improve osteointegration, which requires the formation of bone. ${ }^{[22]}$ Future improvements to the scaffold design would account for this osteoconductive behavior by ensuring improved control of growth factor expression in scaffold regions (i.e., potentially reduced number of transduced human gingival fibroblasts (hGF) cells to lower BMP-7 expression outside of designated bone region), while also redesigning the scaffold construct such that there is direct contact of the scaffold region supporting PDL-like tissue formation with the tooth root.
Immunofluorescence staining provided evidence that the new soft tissue was more mature and PDL-like for the groups with combined patterning and gene delivery. PDL-specific periostin has been shown to positively regulate PDL cell mineralization and differentiation and be involved in maintaining the homeostasis of periodontal tissues. ${ }^{[23]}$ Periostin is a matricellular molecule and mesenchymal stem cell marker that is found in collagen-rich connective tissues, such as tendons and periosteum. At six weeks, the groups with gene delivery showed greater qualitative expression of collagen III and periostin, both markers of ligamentous tissue, throughout the soft tissue region (Figure 4A). However, those groups that
A

Amor+
Empty

Pattern+

Empty

Pattern+

Single

Amor+

Dual

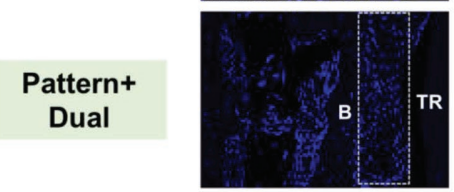

3 WEEKS

B

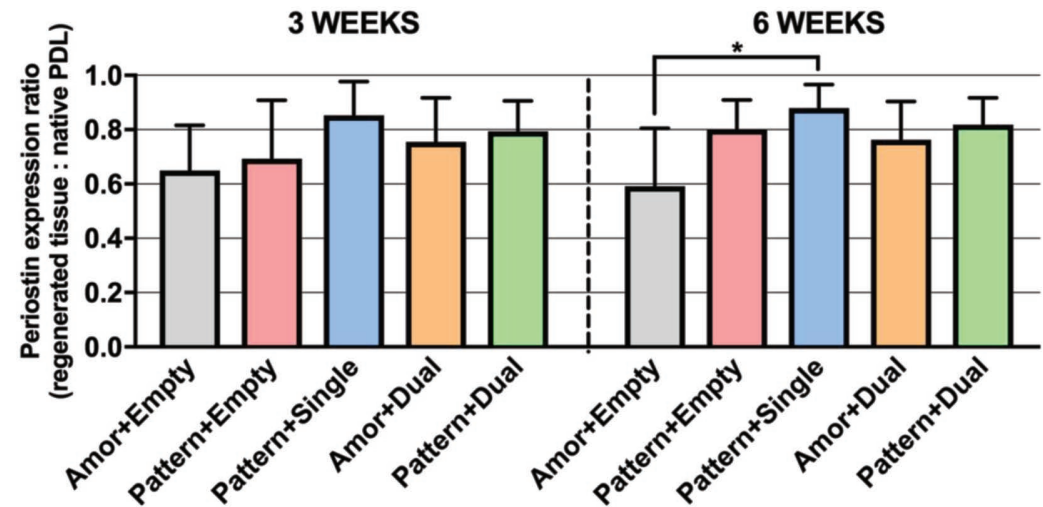

\section{Collagen III}
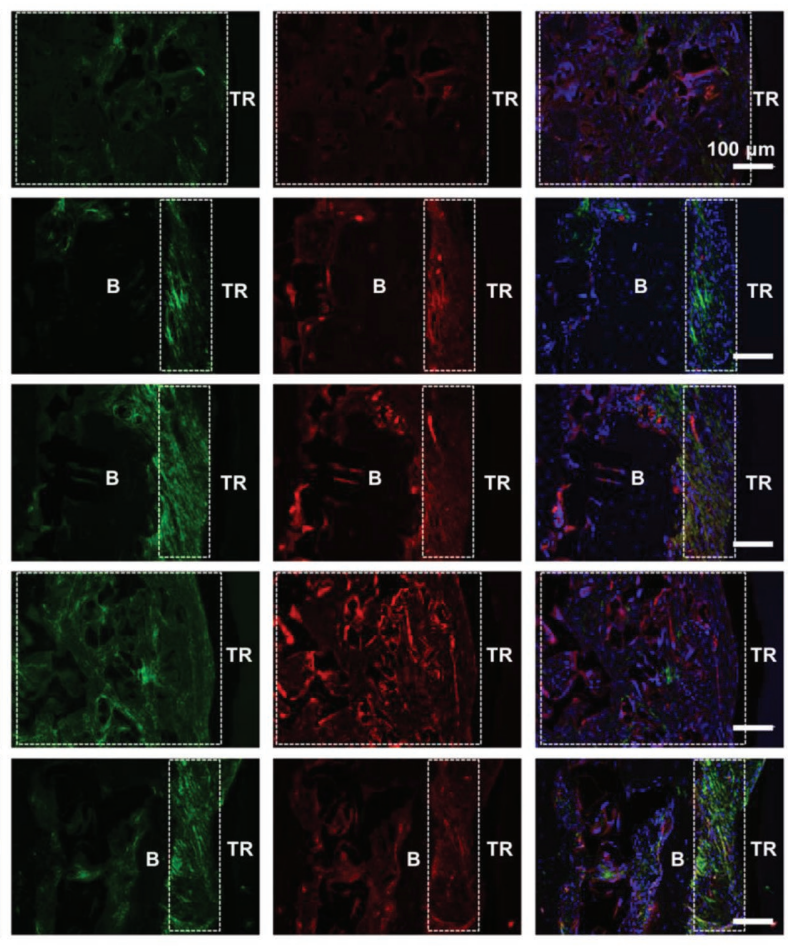
also had patterning (i.e., Pattern+Single and Pattern+Dual) showed more consistent, homogeneous expression, as would be anticipated of mature PDL. Similar, although more modest, results are evident at three weeks (Supporting Information, Figure 2). An analysis of the ratio of mean periostin intensity in the regenerated versus native tissues (or injured (lingual side) versus injured/ regenerated side (buccal side) (Figure 4B) shows significantly higher (albeit modestly increased) expression at three weeks for the Pattern+Single group compared to the Amor+Empty group. Overall, at three weeks the ratios are nonsignificant and lower for the Amor+Empty and Pattern+Empty groups compared to all groups with growth factor delivery. This indicates that the patterning may have a very modest reinforcing effect on PDL-like tissue formation over time, in combination with growth factor expression.

Taken together, these results suggest that, although the combined treatment may not have produced more ligamentous tissue than the other groups, its tissue was more mature and PDL-like. The results of the present study showed that the ratio of periostin expression in regenerated versus native tissues varied little between groups. Surface topography appears to modestly enhance PDL regeneration. In a previous study, combined delivery of AdBMP-7 and AdPDGF via scaffolds contributed to a more robust regeneration of the periodontium than separate delivery of either. ${ }^{[11]}$ Here, however, comparable bone regeneration was observed in groups with single and dual gene delivery, and PDL-like tissue was evident in patterned groups with single and dual delivery.

In human nuclear staining for Amor+Dual and negative control, human-derived cells remained at the defect site even nine weeks postimplantation, more characteristic of an immunodeficient animal model allowing for the longer residence of implanted cells (Figure 5).

\subsection{Mechanical Properties of Tissues, Assessed by Nanoindentation}

Alveolar bone and PDL are load-bearing tissues that integrate over time, making the parameters of stiffness and hardness important for determining whether the regenerated tissue is mechanically similar to its native counterpart. Nanoindentation was done on the experimental group (Pattern+Dual) and negative control (Amor+Empty) after three and nine weeks to evaluate the mechanical properties of the regenerated tissues (Figure 6). These groups were specifically selected in order to

\section{WEEKS}
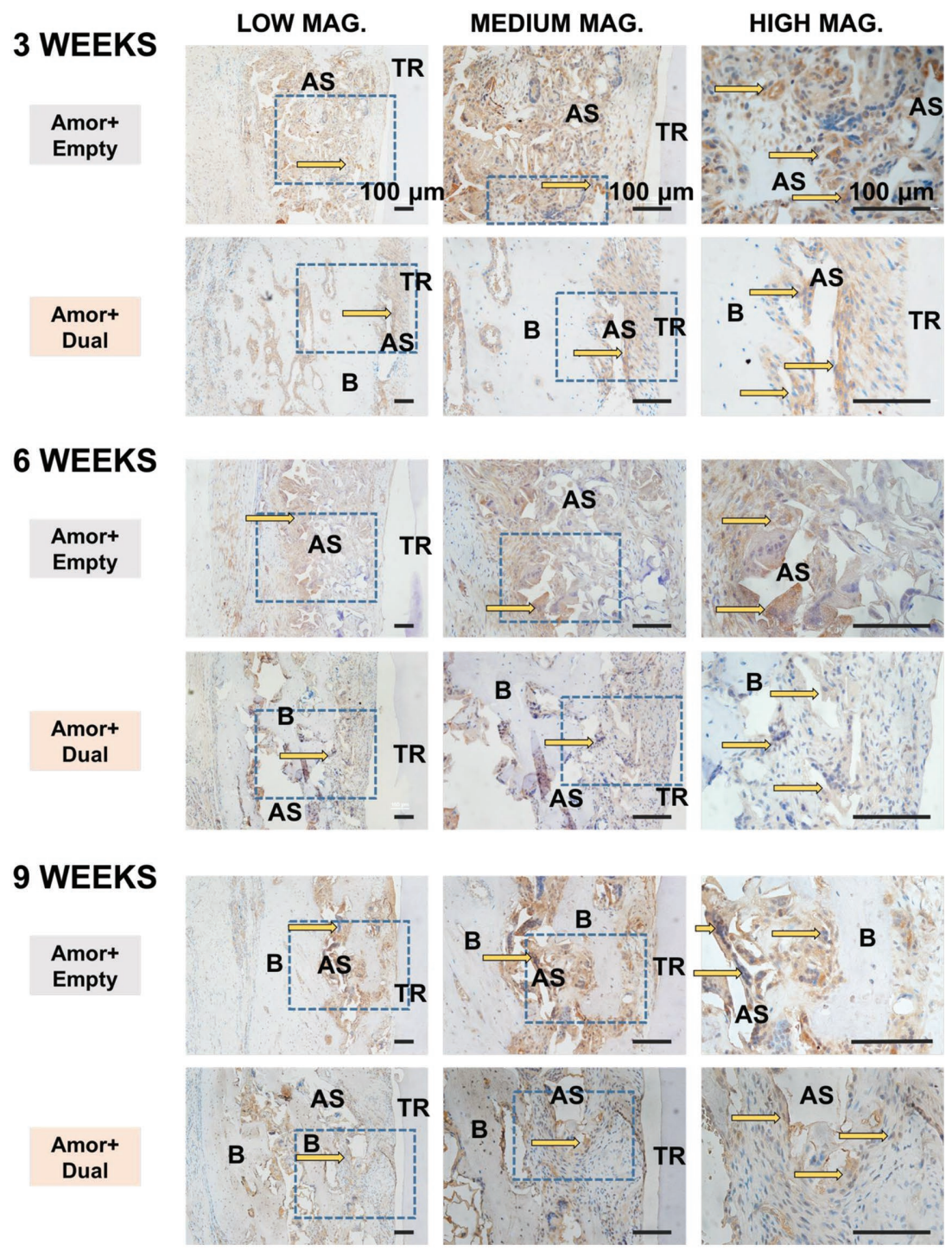

Figure 5. Immunohistochemistry for human cells. Human cells remaining in the defect after 3, 6 , and 9 weeks of healing were stained with antihuman nuclear stain, MACH4, to illustrate the degree to which they contributed to regeneration relative to native rat cells. The remnants of amorphous scaffold take on a nonspecific brown stain; therefore, the human nucleus-positive cells were identified using both the brown stain of MACH4 and blue stain of hematoxylin. Yellow arrows indicate regions with many human cells. Scale bar is $100 \mu \mathrm{m}$ for all images. Boxes indicate the magnified regions of the images to the right. TR = tooth root, $A S=$ amorphous scaffold, $\mathrm{B}=$ bone.

compare and contrast the mechanical properties of the negative and positive controls so as to extract the most relevant findings rather than comparing all groups at once. At the same time, other groups were not taken out to nine weeks to assess tissue regeneration via immunofluorescence, histology, and micro-CT given that the rat fenestration defect model used in this study is known to have a rapid healing rate, giving the expectation that assessments at three and six weeks would suffice to determine the extent of PDL and osseous tissue regeneration, as was found to be true.

The elastic modulus (or stiffness) and hardness were measured for both regenerated and native tissues. Values are reported as ranges due to the heterogeneity of the tissue architecture, as 
A Reduced elastic modulus, $E_{r}(\mathrm{GPa})$ after periodontal regenerative surgery

\begin{tabular}{|c|c|c|c|c|}
\hline 3 WEEKS & $\begin{array}{c}\text { PDL-like } \\
\text { Tissue }\end{array}$ & Regen. Bone & Native PDL & Native Bone \\
\hline Amor+Empty & $\begin{array}{c}0.23-0.79 \\
\text { Pattern+Dual }\end{array}$ & $\begin{array}{c}1.7-3.8 \\
\text { ** }\end{array}$ & $0.18-1.7$ & $\begin{array}{c}1.5-6.9 \\
\star \star\end{array}$ \\
\hline 9 WEEKS & $\begin{array}{c}\text { PDL-like } \\
\text { Tissue }\end{array}$ & $\begin{array}{c}1.5-4.3 \\
\text { Regen. Bone }\end{array}$ & Native PDL & Native Bone \\
\hline Amor+Empty & $\begin{array}{c}0.13-0.72 \\
\#\end{array}$ & $\begin{array}{c}2.5-4.1 \\
\wedge\end{array}$ & $\begin{array}{c}0.22-2.8 \\
\#\end{array}$ & $\begin{array}{c}\text { 2.4-11 } \\
\wedge\end{array}$ \\
\hline Pattern+Dual & $\begin{array}{c}0.24-1.9 \\
2.4-6.3\end{array}$ & \begin{tabular}{c}
$\#$ \\
\hline
\end{tabular}
\end{tabular}

B Hardness, $\boldsymbol{H}(\mathrm{GPa})$ after periodontal regenerative surgery

\begin{tabular}{|c|c|c|c|c|}
\hline 3 WEEKS & $\begin{array}{l}\text { PDL-like } \\
\text { Tissue }\end{array}$ & Regen. Bone & Native PDL & Native Bone \\
\hline Amor+Empty & $\begin{array}{c}0.010-0.030 \\
*\end{array}$ & $0.030-0.15$ & \multirow{2}{*}{$\begin{array}{c}0.010-0.042 \\
\text { * \#\#\# }\end{array}$} & \multirow{2}{*}{$\underset{\wedge \wedge}{0.027-0.17}$} \\
\hline Pattern+Dual & $\begin{array}{c}0.0077-0.020 \\
\text { \#\# }\end{array}$ & $\begin{array}{l}0.022-0.11 \\
\wedge \wedge\end{array}$ & & \\
\hline 9 WEEKS & $\begin{array}{l}\text { PDL-like } \\
\text { Tissue }\end{array}$ & Regen. Bone & Native PDL & Native Bone \\
\hline Amor+Empty & $\underset{\star \star}{0.0057-0.024}$ & $\begin{array}{c}0.070-0.090 \\
\#\end{array}$ & \multirow{2}{*}{$\begin{array}{l}0.01-0.07 \\
\star * \star \star \star \wedge \wedge \wedge \wedge\end{array}$} & \multirow{2}{*}{$\underset{\star \star \star * \star \star \star}{0.07-0.15}$} \\
\hline Pattern+Dual & $\begin{array}{c}0.0049-0.019 \\
\wedge \wedge \wedge \wedge\end{array}$ & 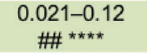 & & \\
\hline
\end{tabular}

C

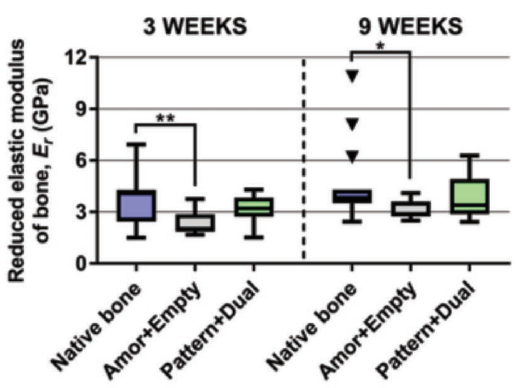

D

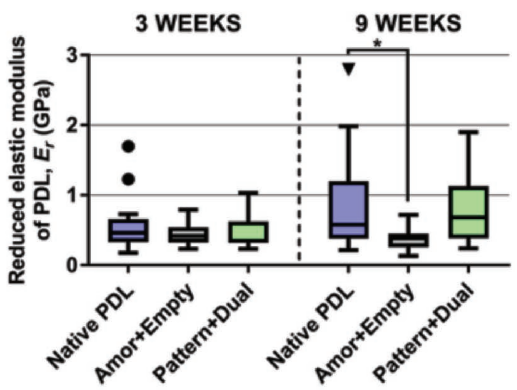

Figure 6. Nanoindentation to assess tissue mechanical properties. A,B) Measurements of the reduced elastic modulus (or stiffness) and hardness of regenerated and native tissues for the experimental group and negative control. Native measurements were taken from uninjured tissue adjacent to the defects and pooled between the two groups. Tukey's box plots compare the stiffness of tissues at C) three weeks and D) nine weeks postimplantation, using data from (A). The regenerated tissues of the negative control are, in 3 of 4 tissues and time points, less stiff than native tissues, while those of the experimental group are statistically indistinguishable from the native tissue. Data shown as ranges for (A) and (B) and as mean \pm SD for (C) and (D). Statistical comparisons were made between the experimental group, negative control, and native tissue for each tissue type at each time point ( $n=15$ per tissue region), using one-way ANOVA with Tukey's post hoc comparisons and two-sided $p$-values of $* / \# / \wedge p<0.05$, $* * p<0.01, * * * p<0.001$, $* * * * p<0.0001$.

has been done previously. ${ }^{[24]}$ At three weeks, the regenerated bone of the negative control was significantly less stiff than native bone (Figure 6C), and at nine weeks, the new bone and PDL-like tissue were less stiff than native (Figure 6A,C,D). By contrast, the stiffness of the experimental group's regenerated tissues was statistically indistinguishable from that of native at three and nine weeks. This suggests greater maturity of the regenerated tissues of the experimental group relative to the control. However, for hardness, both groups' tissues were generally less hard than native, and at nine weeks, the regenerated bone of the experimental group was less hard than that of the negative control (Figure 6B). While elastic modulus property (resistance to elastic deformation in the presence of an applied load) represents the tissue's intrinsic material, hardness (resistance to localized surface deformation) indicates an extrinsic or structurally based response. In general, an increase in both bone tissue stiffness and hardness is expected with increasing time points, with higher ranges for PDL and bone tissues in the experimental group relative to the negative control. The increase in stiffness of bone and PDL tissues in the experimental group relative to the negative control group is observed, while the hardness range is shown to be on the lower end for the experimental group relative to the negative control group. Presence of increased tissue mineralization in negative control samples can increase tissue hardness, which is a possibility given that the negative control samples may be at risk of ankylosis-like formations as a result of regenerative responses (without intervening PDL soft vs osseous tissue formation) that could be verified histologically over longer periods of time than what was observed in this study at weeks 3 and 6. Additionally, it is worthwhile to note that the resulting PDL-like tissue mechanical properties are also affected by the lack of integration between PDL tissue and regenerated bone, as is the case in native periodontium, thereby changing the stiffness and hardness of these tested regenerated PDL samples when compared to native PDL tissues. The histological samples reveal that the soft PDL-like tissues do not have any defined points of integration, instead showing distinct areas of either soft or osseous tissue formation. Potential changes to the scaffold design that results in a more integrated scaffold to begin with instead of two separate regions, may contribute to a more natural integration between these two tissue types over the course of the regenerative process.

Overall, the values observed for the native bone and PDL here correspond to those of other studies. ${ }^{[24,25]}$ That said, it is important to note that a variety of technical factors may affect the stiffness and hardness ranges, including the hydration state (i.e., testing under wet vs dry conditions), probe geometry, and loading conditions. Moreover, the heterogeneity of values in the bone tissue is specifically identifiable using nanoindentation, as compared with microindentation, given that there are heterogeneous regions of lamellar bone integrating with bundle bone, thereby resulting in a range of values. On the whole, the nanoindentation data indicate that, with respect to stiffness, the experimental group more closely approximated the mechanical properties of native tissue than the negative control, but that both tissues had not yet reached full maturity, as shown by hardness. 


\section{Conclusions}

This study aimed to evaluate the effects of combining surface topography with growth factor gene delivery on periodontal tissue regeneration. The results indicate that the combination better regenerates the bone-PDL interface than either treatment alone. Delivery of growth factor genes, principally AdPDGF-BB and AdBMP-7, improves bone formation, and surface topography incorporated into the scaffold as micropatterning was important for the formation of PDL-like tissue and the overall integrity of the bone-PDL interface. This study adds nuance to strategies that use biomaterials for regenerative treatments for periodontal and other diseases. It also adds to previous knowledge that micropatterning can enhance tissue responses, and that gene therapy vectors can be immobilized onto a scaffold surface to localize the transduction of cells in vivo. Future work is needed to calibrate the effects of scaffold topography to activate the appropriate cellular responses for regenerating specific tissue types, as well as develop a process for scaffold fabrication that permits transduction to occur in vivo instead of in vitro prior to implantation.

\section{Experimental Section}

Preparation of Biphasic Scaffolds with Micropatterned and Amorphous Regions: A CAD-based program (Tanner L-Edit IC, Mentor, Siemens, Munich, Germany) was used to design the micropatterns of the scaffolds and master molds with the parameters necessary for an alveolar bone defect. Design specifications for the micropatterns consisted of the following: pillar height of $100 \mu \mathrm{m}$, interpillar distance of $150 \mu \mathrm{m}$, pillar length $\times$ width of $150 \mu \mathrm{m} \times 150 \mu \mathrm{m}$, and grooves of $15 \mu \mathrm{m}$ width $\times 30 \mu \mathrm{m}$ depth (Figure 1B). The pillar height approximated the thickness of native rat PDL as identified in pilot studies (data not shown). SU-8 master molds were fabricated at Lurie Nanofabrication Facility (University of Michigan, Ann Arbor, MI) using standard photolithography as previously described. ${ }^{[7]}$ Briefly, polydimethylsiloxane (PDMS, Sylgard 184, Dow Corning, Midland, MI) was mixed in a ratio of 10:1 v/v of base to curing agent, degassed under vacuum, poured onto SU-8 master molds, cured at $65{ }^{\circ} \mathrm{C}$, and peeled to reveal the pattern.

Fabrication of the PDL Region: A solution of $5 \%$ poly(D,L-lactic-coglycolic acid) (75:25 lactic:glycolic, $137 \mathrm{kDa}$, Evonik Industries, Essen, Germany) and polycaprolactone (45 kDa, Sigma-Aldrich, St. Louis, MO) in a 1:1 ratio by weight was used to cast the pillars within a PDMS mold. To complete the PDL region, a solution of $10 \%$ PCL was layered onto the precast pillars while adding sugar particles $(\leq 75 \mu \mathrm{m})$, in order to create a base of $150 \mu \mathrm{m}$ thickness. After drying under vacuum overnight, the sugar was leached out in deionized water, leaving porosity for cell growth.

Fabrication of the Bone Region: A porous, amorphous PCL scaffold was formed in a similar manner as for the PDL region's base, with a final thickness of $250 \mu \mathrm{m}$, such that the combined biphasic scaffold construct could fit within a $0.5 \mathrm{~mm}$ deep fenestration defect.

In order to test the effect of topography on PDL regeneration, an amorphous, porous PCL/PLGA (1:1 by weight) PDL region with a total thickness of $250 \mu \mathrm{m}$ was formed using the same technique as the bone region. This monophasic scaffold represented the unpatterned control.

CVD and Gene Therapy Vector Immobilization: Following scaffold fabrication, each region of the scaffold was separately coated with a layer of amine-reactive polymer using a custom-built CVD system, as previously reported. ${ }^{[14,17]}$ Briefly, the scaffolds were fixed inside the deposition chamber at $15{ }^{\circ} \mathrm{C}$, and the pentafluorophenyl estersubstituted paracyclophane dimer was sublimated at $120^{\circ} \mathrm{C}$, pyrolyzed at $540{ }^{\circ} \mathrm{C}$ to form a stream of reactive diradical vapor, and deposited and polymerized on the scaffold surface (pressure of $0.1 \mathrm{mbar}$, with argon as the carrier gas). The scaffolds were then turned over and coated again to ensure that the coating deposited on all sides. The scaffolds were stored under vacuum until further use or sterilized in $70 \%$ ethanol to prepare for adenoviral immobilization onto their surfaces, as needed.

For gene immobilization, scaffolds were treated with anti-adenovirus antibodies and adenoviruses, as previously reported. ${ }^{[14]}$ Briefly, after sterilization, scaffolds were incubated with $10 \mu \mathrm{g} \mathrm{mL} \mathrm{m}^{-1}$ goat antiadenovirus polyclonal antibody (0151-9004, AbD Serotec, Bio-Rad Laboratories, Hercules, CA) in phosphate-buffered saline (PBS) at $4{ }^{\circ} \mathrm{C}$ overnight, based on a previously established saturation point. They were then rinsed $5 \times 5 \mathrm{~min}$ with PBS, incubated in $12 \mathrm{~mL}$ of $10^{12}$ particles (PN) $\mathrm{mL}^{-1}$ cold adenovirus solution at $4{ }^{\circ} \mathrm{C}$ for $4 \mathrm{~h}$ and rinsed $5 \times 5 \mathrm{~min}$ with PBS. After the final PBS wash, the scaffolds were ready for cell seeding.

Replication-deficient adenoviruses containing the genes of interest were prepared by the University of Michigan Vector Core. During the adenoviral incubation, experimental scaffolds received AdBMP-7 for their bone regions (i.e., amorphous PCL scaffold) and AdPDGF-BB or BMP-7 for their PDL regions (i.e., porous micropatterned PLGA film) (Figure 1A). Control groups received adenoviruses encoding nonsense vectors (Ad-empty) for their bone and PDL regions.

Cell Seeding: hPDLs (primary cells from PDL, No. 2630, ScienCell, Carlsbad, CA) were grown in Dulbecco's Modified Eagle Media (DMEM, Thermo Fisher, Ann Arbor, MI) with 5\% fetal bovine serum (FBS) at $37^{\circ} \mathrm{C}$. hGFs (primary cells from gingiva, No. 2620, ScienCell) were grown in DMEM with $10 \% \mathrm{FBS}$ at $37^{\circ} \mathrm{C}$. The day prior to surgical implantation, $5 \times 10^{5} \mathrm{hPDLs}$ were seeded onto the PDL regions of the scaffolds (except the single-gene delivery group, for which hGFs were seeded onto the patterned, AdBMP-7-coated PDL region), and $5 \times 10^{5} \mathrm{hGFs}$ were seeded onto the bone regions. The cells were allowed to attach before transferring the scaffolds into fresh medium and incubating at $37^{\circ} \mathrm{C}$ until surgery. The selection of hGFs for seeding of the bone region, instead of human osteoblasts, was based on the fact that these cells are more easily accessible clinically and that the transduction of these cells with Ad-BMP7 results in formation of bone that occurs more rapidly and in similar bone formation as would be expected with the use of human osteoblasts alone. At the same time, this approach is intended to provide greater control over the location of bone formation within the scaffold region by immobilizing the vector through CVD-based coating onto polymer surface in regions of interest, instead of randomly seeding osteoblasts without more spatiotemporal control over at least the initial expression of BMP7 in the scaffold.

Scanning Electron Microscopy: CVD-coated scaffolds with and without hPDL seeding were imaged by SEM. Those with cells were prepared for imaging $3 \mathrm{~d}$ after seeding by fixing with $2.5 \%$ glutaraldehyde at $4{ }^{\circ} \mathrm{C}$ overnight, dehydrating with ethanol, and washing three times with hexamethyldisilazane (HMDS). Scaffolds were mounted using graphite conductive adhesive (No. 12691-30, Electron Microscopy Sciences, Hatfield, PA), gold-sputter coated, and observed at an acceleration voltage of $5 \mathrm{kV}$ using an AMRAY FE 1900 SEM (SEMTech Solutions, Billerica, MA) at the Microscopy and Image Analysis Laboratory (University of Michigan).

Barium Sulfate Treatment: To visualize a scaffold's adaptation to the fenestration defect, a full scaffold (i.e., both the micropatterned PDL and bone regions) was immersed in $20 \%$ barium sulfate in water for $30 \mathrm{~min}$, prior to being placed into a rat fenestration defect $(3 \times 2 \mathrm{~mm})$ on a defleshed, cadaver mandible and scanned using micro-CT, as detailed below.

In Vivo Implantation: Rat Fenestration Defect: The in vivo study design consisted of the following five groups, all CVD-coated and cellseeded: (1) a negative control with Ad-empty, amorphous PDL region and Ad-empty bone region (Amor+Empty); (2) Ad-empty, patterned PDL and Ad-empty bone (Pattern+Empty); (3) AdBMP-7, patterned PDL and AdBMP-7 bone (with hGF cells in both, Pattern+Single); (4) AdPDGF-BB, amorphous PDL and AdBMP-7 bone (Amor+Dual); and (5) the experimental treatment with AdPDGF-BB, patterned PDL and AdBMP-7 bone (Pattern+Dual) (Figure 1A). The time points that tested 
tissue regeneration were at three and six weeks. For the three-week time point, Amor+Empty and Pattern+Dual had a total $n=10$, and the remaining groups (Pattern+Empty, Pattern+Single, Amor+Dual) had $n=$ 6 because these groups were not used for additional nanoindentation assessments. For the six-week time point, all groups had $n=6$ to allow for micro-CT, immunofluorescence, and histological assessments. Amor+Empty, Amor+Dual, and Pattern+Dual were also extended to nine weeks $(n=5)$ to evaluate the retention of human-based cells and the mechanical properties of regenerated bone and PDL-like tissue compared to native tissues.

All animal procedures were performed with approval of the University of Michigan-University Committee on Use and Care of Animals (UM-UCUCA) according to ARRIVE guidelines for preclinical studies. Athymic male rats $(250 \mathrm{~g}$, Charles River Laboratories, Wilmington, MA) were ordered, administered preemptive subcutaneous analgesic (5 $\mathrm{mg} \mathrm{kg}^{-1}$ carprofen), and put under general anesthesia with isofluorane during periodontal defect creation. A single fenestration defect $(3 \mathrm{~mm}$ width $\times 2 \mathrm{~mm}$ height) was created for each animal on the buccal side of the right mandible, such that the distal root of the first molar tooth was exposed (Supporting Information, Figure 1). The cementum layer was carefully removed to expose the dentin surface, after which the PDL region of the scaffolds was positioned with the pillars facing the dentin, followed by the bone region directly adjacent. The site was closed with sutures and surgical staples, with subcutaneous administration of analgesic $24 \mathrm{~h}$ postsurgery. After 3, 6, and 9 weeks, specimens were harvested and fixed in $10 \%$ buffered formalin phosphate solution for $2 \mathrm{~d}$ before being transferred into $70 \%$ ethanol for micro-CT scanning.

Micro-CT and Histomorphometry: Micro-CT was used to evaluate bone regeneration postimplantation and scaffold adaptation. Tissuefixed specimens were embedded in alginate, scanned using a $\mu C T$ 100 cabinet micro-CT scanner (Scanco Medical, Brüttisellen, Switzerland) at a resolution of $12 \mu \mathrm{m}, 70 \mathrm{kV}$ energy, and $114 \mu \mathrm{A}$ intensity, and calibrated to Hounsfield units (HU). Total bone volume, bone fill percent, and TMD were determined for regenerated bone in the healed defects using Microview (Parallax Innovations, Ilderton, Ontario, Canada) at a threshold of $\mathrm{HU}=1050$ for bone ( $n=6-10$ per group). After scanning, samples were decalcified in 10\% EDTA, embedded in paraffin, and cut into $5 \mu \mathrm{m}$ sections for histological analysis.

Some sections were stained with toluidine blue for histomorphometric analysis. Soft tissue width was determined by measuring from the scaffold or newly formed bone to the tooth root at three points on each sample: near the two edges of the scaffold (at the farthest pillar, if applicable) and at its center; these three measurements were then averaged for the sample (Figure $3 \mathrm{~B})(n=3-6$ samples, depending on the group and time point)

Other sections were stained with H\&E to evaluate soft tissue formation. Some of those also underwent human nuclear staining performed using immunohistochemistry. The $5 \mu \mathrm{m}$ sections were dewaxed and incubated in DIVA antigen retrieval solution (Biocare Medical, Concord, CA) at $60{ }^{\circ} \mathrm{C}$ overnight. Next, they were incubated with $3 \%$ bovine serum albumin (BSA) for blocking of unspecific binding followed by incubation overnight at $4{ }^{\circ} \mathrm{C}$ with primary antibody (mouse antihuman nuclei monoclonal antibody, diluted 1:100, MAB1281, EMD Millipore Corp, Temecula, CA), overnight at $4{ }^{\circ} \mathrm{C}$. The sections were then incubated with $\mathrm{MACH} 4$ polymer (Biocare Medical) for $30 \mathrm{~min}$. Positive cells were detected using DAB substrate (Biocare Medical) plus the H\&E counterstain.

Immunofluorescence: Sections of $5 \mu \mathrm{m}$ thickness were dewaxed, rehydrated, and incubated in DIVA antigen retrieval solution (Biocare Medical) at $60{ }^{\circ} \mathrm{C}$ overnight ( $n=6$ for all groups). Following blocking of unspecific binding using $3 \% \mathrm{BSA}$, the sections were incubated at $4{ }^{\circ} \mathrm{C}$ overnight with primary antibodies for anti-periostin (rabbit polyclonal, ab14041, Abcam, Cambridge, MA) and anti-collagen III (mouse monoclonal, ab6310, Abcam) at 1:500 dilutions. The sections were then incubated with Alexa-conjugated secondary antibodies at a 1:200 dilution for $2 \mathrm{~h}$ at room temperature (Alexa-488 anti-mouse, ab150113, and Alexa-555 anti-rabbit, ab150074, Abcam). Controls did not use the primary antibody in the protocol. The comparison of antibody staining for periostin contrasted the buccal (injured and/or regenerated bone defect area) versus the lingual (uninjured, nonsurgically treated bone). 4',6-Diamidino-2-phenylindole (DAPI) was additionally used to visualize cell nuclei (Prolong Gold Antifade Reagent with DAPI, Life Technologies, Carlsbad, CA).

Nanoindentation: Mandibulae reserved for nanoindentation were frozen after harvesting at three and nine weeks postimplantation. Samples were prepared for mechanical testing as follows: thawing the frozen sample, trimming the mandible to retain only the molars by cutting off the incisors and ramus using a low-speed diamond wheel saw (South Bay Technology, San Clemente, CA), embedding the trimmed sample in epoxy resin (EMBED 812 Embedding Kit, Electron Microscopy Sciences, Hatfield, PA), sectioning using a microtome (Leica Biosystems, Wetzlar, Germany), polishing using silicon carbide abrasive paper (2400 grit) under water irrigation for $2 \mathrm{~min}$, and cleansing to remove particles using ultrasonication in water for $15 \mathrm{~min}$.

Nanoindentation was performed using the $950 \mathrm{TI}$ Tribolndenter (Hysitron Incorporated, Minneapolis, MN) at the Michigan Center for Materials Characterization ( $\mathrm{MC}^{2}$, University of Michigan). The sample was glued to a steel stub (Ted Pella, Altadena, CA) and indented using a standard Berkovich diamond probe with a penetration depth of $500 \mathrm{~nm}$ and $3 \mathrm{~s}$ for each load, hold, and unload. ${ }^{[24,25]}$ Fused silica was used as the calibration standard. The reduced modulus $\left(E_{\mathrm{r}}\right)$ and hardness $(H)$ were calculated using the load-displacement curve based on five indents (each $10 \mu \mathrm{m}$ apart, in accordance with ASTM standards) at the following four regions for each sample: (1) regenerated PDL-like tissue at the original defect site, (2) regenerated alveolar bone, (3) native alveolar bone at the region directly opposite the defect site, and (4) native PDL ( $n=3$ samples $\times 5$ indentations for each tissue region).

Specifically, the $E_{\mathrm{r}}(\mathrm{GPa})$ is calculated based on Oliver-Pharr analysis using Equation (1), where $S\left(\mu \mathrm{N} \mathrm{nm}^{-1}\right)$ is the contact stiffness (obtained from the unload curve slope, where $P$ is the indentation force and $h$ is displacement), and $A$ is the projected area $\left(\mathrm{nm}^{2}\right)$ of elastic contact

$E_{\mathrm{r}}=\frac{\sqrt{\pi}}{2} \frac{S}{\sqrt{A}}$, where $S=\left.\frac{\mathrm{d} P}{\mathrm{~d} h}\right|_{P_{\max }}$

The hardness, $H(\mathrm{MPa})$, is calculated using Equation (2), where $P_{\max }$ is the maximum indentation force $(\mu N)$ and $A_{C}$ is the projected area $\left(\mathrm{nm}^{2}\right)$ of contact

$H=\frac{P_{\max }}{A_{C}}$

Statistical Analysis: Data were expressed as mean \pm standard deviation for all graphs, save for the tables in Figure 6 where ranges are shown. Using GraphPad Prism, one-way analysis of variance (ANOVA) with post hoc Tukey's multiple comparison method was used to perform comparative analysis within each time point and a two-sided $p$-value of less than $0.05(\alpha<0.05)$ considered significant. Levels of significance are indicated in the figure legends. Sample sizes for the analyses are as follows: $n=6-10$ depending on the group and time point for bone volume, bone fill percent, and tissue mineral density; $n=6$ for periostin expression ratio; $n=3-6$ for soft tissue width; $n=15$ for nanoindentation ( 3 samples $\times 5$ indentations per tissue type).

\section{Supporting Information}

Supporting Information is available from the Wiley Online Library or from the author.

\section{Acknowledgements}

The authors would like to thank the following individuals for their assistance during this study: Dr. Richard Miron for his surgical assistance of the animal model, to Sarah Volk, Jonathan Oh, Allyson 
Christman, Dr. Qiming Jin, Chris Strayhorn (University of Michigan School of Dentistry Histology Core), and Michelle Lynch (University of Michigan School of Dentistry Micro-CT Core). This study was supported by the National Institutes of Health/Nationallnstitute for Dental and Craniofacial Research (NIH/NIDCR DE 13397 and U24 DE026915) to W.V.G., the NIH/NIDCR Tissue Engineering at Michigan (TEAM) trainee grant (DE 007057), the National Science Foundation Graduate Research Fellowship Program (1256260), the University of Michigan Rackham Merit Fellowship to S.P.P., and the Osteology Foundation Research Scholarship to T.F.

\section{Conflict of Interest}

The authors declare no conflict of interest.

\section{Keywords}

gene therapy, micropatterning, periodontal regeneration, platelet-derived growth factor (PDGF), scaffolds

Received: June 29, 2018

Revised: September 4, 2018

Published online: October 19, 2018

[1] J. Lindhe, Textbook of Clinical Periodontology, Munksgaard, Copenhagen, Denmark 1983.

[2] a) C. H. Park, H. F. Rios, Q. Jin, M. E. Bland, C. L. Flanagan, S. J. Hollister, W. V. Giannobile, Biomaterials 2010, 31, 5945; b) C. H. Park, H. F. Rios, Q. Jin, J. V. Sugai, M. Padial-Molina, A. D. Taut, C. L. Flanagan, S. J. Hollister, W. V. Giannobile, Biomaterials 2012, 33, 137; c) C. H. Park, H. F. Rios, A. D. Taut, M. Padial-Molina, C. L. Flanagan, S. P. Pilipchuk, S. J. Hollister, W. V. Giannobile, Tissue Eng., Part C 2014, 20, 533; d) S. Ivanovski, C. Vaquette, S. Gronthos, D. W. Hutmacher, P. M. Bartold, J. Dent. Res. 2014, 93, 1212; e) C. Vaquette, W. Fan, Y. Xiao, S. Hamlet, D. W. Hutmacher, S. Ivanovski, Biomaterials 2012, 33, 5560.

[3] L. Larsson, S. P. Pilipchuk, W. V. Giannobile, R. M. Castilho, J. Biomed. Mater. Res., Part B 2018, 106, 2065.

[4] M. Köunönen, M. Hormia, J. Kivilahti, J. Hautaniemi, I. Thesleff, J. Biomed. Mater. Res. 1992, 26, 1325.

[5] P. Lagonegro, G. Trevisi, L. Nasi, L. Parisi, E. Manfredi, S. Lumetti, F. Rossi, G. M. Macaluso, G. Salviati, C. Galli, Dent. Mater. J. 2018, $37,278$.

[6] a) F. S. M. Ismail, R. Rohanizadeh, S. Atwa, R. S. Mason, A. J. Ruys, P. J. Martin, A. Bendavid, J. Mater. Sci.: Mater. Med. 2007, 18, 705; b) J. Chen, S. Mwenifumbo, C. Langhammer, J. P. McGovern, M. Li, A. Beye, W. O. Soboyejo, J. Biomed. Mater. Res., Part B 2007, 82B, 360.
[7] S. P. Pilipchuk, A. Monje, Y. Jiao, J. Hao, L. Kruger, C. L. Flanagan, S. J. Hollister, W. V. Giannobile, Adv. Healthcare Mater. 2016, 5, 676.

[8] D. Kaigler, J. A. Cirelli, W. V. Giannobile, Expert Opin. Drug Delivery 2006, 3, 647.

[9] M. Nevins, R. T. Kao, M. K. McGuire, P. K. McClain, J. E. Hinrichs, B. S. McAllister, M. S. Reddy, M. L. Nevins, R. J. Genco, S. E. Lynch, W. V. Giannobile, J. Periodontol. 2013, 84, 456.

[10] a) P.-C. Chang, J. A. Cirelli, Q. Jin, Y.-J. Seol, J. V. Sugai, N. J. D'Silva, T. E. Danciu, L. A. Chandler, B. A. Sosnowski, W. V. Giannobile, Hum. Gene Ther. 2009, 20, 486; b) Q. Jin, O. Anusaksathien, S. A. Webb, M. A. Printz, W. V. Giannobile, Mol. Ther. 2004, 9, 519; c) Q. M. Jin, O. Anusaksathien, S. A. Webb, R. B. Rutherford, W. V. Giannobile, J. Periodontol. 2003, 74, 202.

[11] Y. Zhang, J. M. Richard, S. Li, B. Shi, A. Sculean, X. Cheng, J. Clin. Periodontol. 2015, 42, 262.

[12] Y. Zhang, B. Shi, C. Li, Y. Wang, Y. Chen, W. Zhang, T. Luo, X. Cheng, J. Controlled Release 2009, 136, 172.

[13] J. Lahann, D. Klee, W. Pluester, H. Hoecker, Biomaterials 2001, 22, 817.

[14] J. Hao, C. K. C. Kenneth, G. K. Laura, L. Larsson, V. S. James, J. Lahann, V. G. William, Adv. Mater. 2016, 28, 3145.

[15] W. J. King, P. H. Krebsbach, Adv. Drug Delivery Rev. 2012, 64, 1239.

[16] Y. M. Elkasabi, J. Lahann, P. H. Krebsbach, Biomaterials 2011, 32, 1809.

[17] Y. Zhang, X. Deng, E. L. Scheller, T.-G. Kwon, J. Lahann, R. T. Franceschi, P. H. Krebsbach, Biomaterials 2010, 31, 3231.

[18] R. T. Franceschi, D. Wang, P. H. Krebsbach, R. B. Rutherford, J. Cell. Biochem. 2000, 78, 476.

[19] W.-W. Hu, Y. Elkasabi, H.-Y. Chen, Y. Zhang, J. Lahann, S. J. Hollister, P. H. Krebsbach, Biomaterials 2009, 30, 5785 .

[20] S. Sowmya, U. Mony, P. Jayachandran, S. Reshma, R. A. Kumar, H. Arzate, V. N. Shantikumar, R. Jayakumar, Adv. Healthcare Mater. 2017, 6, 1601251

[21] M. de Wild, S. Zimmermann, J. Ruegg, R. Schumacher, T. Fleischmann, C. Ghayor, F. E. Weber, 3D Print. Addit. Manuf. 2016, 3, 142.

[22] L. Lin, H. Wang, M. Ni, Y. Rui, T.-Y. Cheng, C.-K. Cheng, X. Pan, G. Li, C. Lin, J. Orthop. Transl. 2014, 2, 35.

[23] a) S. Yamada, T. Tauchi, T. Awata, K. Maeda, T. Kajikawa, M. Yanagita, S. Murakami, J. Dent. Res. 2014, 93, 891; b) H. F. Rios, L. F. Bonewald, S. J. Conway, J. Dent. Res. 2014, 93, 843.

[24] a) S. P. Ho, M. P. Kurylo, T. K. Fong, S. S. J. Lee, H. D. Wagner, M. I. Ryder, G. W. Marshall, Biomaterials 2010, 31, 6635; b) S. P. Ho, M. P. Kurylo, K. Grandfield, J. Hurng, R.-P. Herber, M. I. Ryder, V. Altoe, S. Aloni, J. Q. Feng, S. Webb, G. W. Marshall, D. Curtis, J. C. Andrews, P. Pianetta, Bone 2013, 57, 455; c) S. P. Ho, S. J. Marshall, M. I. Ryder, G. W. Marshall, Biomaterials 2007, 28, 5238.

[25] K. Grandfield, R.-P. Herber, L. Chen, S. Djomehri, C. Tam, J.-H. Lee, E. Brown, W. R. Woolwine, D. Curtis, M. Ryder, J. Schuck, S. Webb, W. Landis, S. P. Ho, Bone Rep. 2015, 3, 20. 